Regular Article

\title{
Experimental and theoretical modelling of blind-ended vessels within a developing angiogenic plexus
}

\author{
Luciano A. Guerreiro-Lucas ${ }^{\mathrm{a}, 1}$, S. Rares Pop ${ }^{\mathrm{b}, 1,2}$, Maria J.C. Machado a , Ying Liang Ma ${ }^{\text {a }}$, Sarah L. Waters ${ }^{\mathrm{b}, 3}$, \\ Giles Richardson $^{\text {b }}$, Kurt Saetzler ${ }^{a}$, Oliver E. Jensen ${ }^{\text {b,* }}$, Christopher A. Mitchell ${ }^{\mathrm{a}, *}$ \\ a Centre for Molecular Biosciences, School of Biomedical Sciences, University of Ulster, Cromore Road, Coleraine, BT52 1SA, UK \\ b School of Mathematical Sciences, University of Nottingham, University Park, Nottingham, NG7 2RD, UK
}

\section{A R T I C L E I N F O}

\section{Article history:}

Received 15 January 2008

Revised 30 May 2008

Accepted 27 June 2008

Available online $\mathrm{xxxx}$

\section{Keywords:}

Blind-ended vessels

Wound healing

Angiogenesis

Intra-vital microscopy

Mathematical modelling

Longitudinal study

\begin{abstract}
A B S T R A C T
Angiogenic sprouts at the leading edge of an expanding vascular plexus are recognised as major regulators of the structure of the developing network. Early in sprout development, a vascular lumen is often evident which communicates with the parent vessel while the distal tip is blind-ended. Here we describe the temporal evolution of blind-ended vessels (BEVs) in a small wound made in the panniculus carnosus muscle of a mouse viewed in a dorsal skin-fold window-chamber model with intra-vital microscopy during the most active period of angiogenesis (days 5-8 after injury). Although these structures have been mentioned anecdotally in previous studies, we observed BEVs to be frequent, albeit transient, features of plexus formation. Plasma leakage into the surrounding extracellular matrix occurring from these immature conduits could play an important role in preparing hypoxic tissue for vascular invasion. Although sprout growth is likely to be regulated by its flow environment, the parameters regulating flow into and through BEVs have not been characterised in situ. Longitudinal data from individual animals show that the number of BEVs filled with plasma alone peaks at day 7, when they can exceed $150 \mu \mathrm{m}$ in length. Additionally, BEVs greater than $40 \mu \mathrm{m}$ in length are more likely to be filled with stationary erythrocytes than with plasma alone. Using a mathematical model, we show how the flux of $150 \mathrm{kD}$ fluorinated (FITC-) dextran through an individual plasma-filled BEV is related to its geometry being determined primarily by its surface area; by fitting theoretical intensity values to experimental data we assess the permeability of the vessel to FITC-dextran. Plasma skimming provides a mechanistic explanation for the observation that BEVs with larger surface area are more likely to recruit erythrocytes.
\end{abstract}

(c) 2008 Elsevier Inc. All rights reserved.

\section{Introduction}

Establishment of a circulatory system is a key stage in the embryonic growth of vertebrates, which allows organ growth and the subsequent differentiation of tissue types. The maintenance of an appropriate vascular supply in the adult is a tightly regulated process, which is often temporally restricted. This is particularly evident in the hormonally-driven cyclical control of vascular growth in the female reproductive organs (particularly the ovary, corpus luteum and uterus) during the menstrual cycle, as well as in the intense vascular

\footnotetext{
* Corresponding authors. Jensen O.E. is to be contacted at fax: +44 1159513837. Mitchell C.A., fax: +44 2870324375

E-mail addresses: Oliver.Jensen@nottingham.ac.uk (O.E. Jensen), ca.mitchell@ulster.ac.uk (C.A. Mitchell).

1 These authors contributed equally to this manuscript.

2 Current address: School of Computer Science, Bangor University, Dean Street, Bangor, Gwynedd, LL57 1UT, UK.

${ }^{3}$ Current address: Oxford Centre for Industrial and Applied Mathematics, Mathematical Institute, 24-29 St. Giles', Oxford, OX1 3LB, UK.
}

growth and remodelling that occur during physiological wound healing.

There are at least 5 distinct morphological processes in which vascular plexi are established, maintained or their structural complexity is increased: vasculogenesis (Risau and Flamme, 1995), angiogenesis (Carmeliet and Jain, 2000), intussusception (Burri, 1992), elongation (Gargett and Rogers, 2001) and integration of circulating endothelial precursors (Kopp et al., 2006). Although vasculogenesis is largely restricted to embryogenesis and extra-embryonic tissues (such as the yolk-sac and placenta), all of the other processes have been described in either normal physiological or pathological conditions in the adult. The most intensively studied of these processes is angiogenesis, whose defining characteristic is the initial sprouting of solid vessel cords from pre-existing vasculature.

Angiogenesis is a multi-step process, starting with the activation of endothelial cells (ECs) by a local preponderance of pro-angiogenic factors over inhibitors, previously described as the angiogenic switch (Hanahan and Folkman, 1996). Activated ECs loosen their homotypic interactions, produce extracellular matrix-degrading enzymes and migrate towards the angiogenic stimulus. Specialised endothelial "tip 

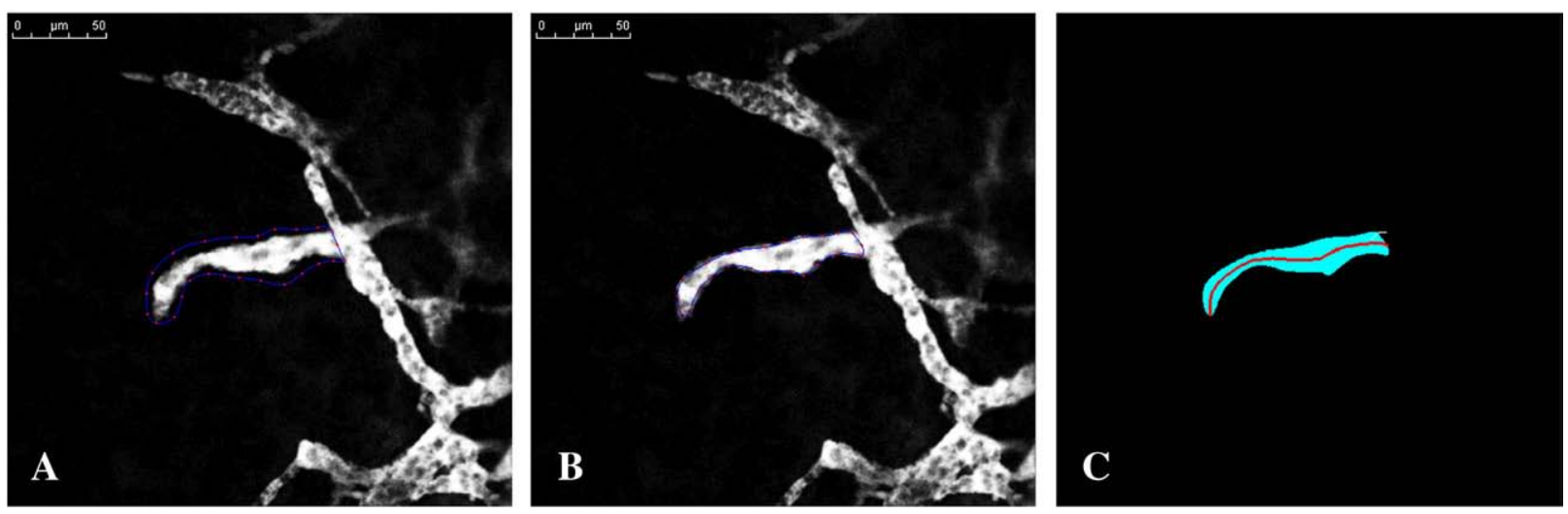

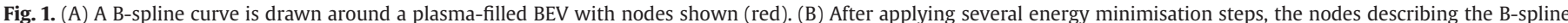

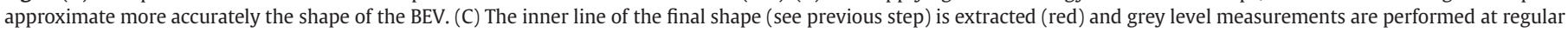
intervals along this inner line. (For interpretation of the references to colour in this figure legend, the reader is referred to the web version of this article.)

cells" direct the advancing cord and the cells at the base of this structure are able to proliferate, providing a pool of cells for expansion of the growing sprout (Gerhardt, 2003). These solid EC cords initially develop a lumen, forming blind-ended vessels; the tips of these vessels may join with other segments, establishing blood flow through the loop, as recently shown in the zebrafish gastrula (Vogeli, 2006). The transport of oxygen and nutrients though these newly formed vessels is mediated by well-characterised non-linear rheological effects (such as the plasma-skimming, Fahraeus and FahraeusLindqvist effects (Popel and Johnson, 2005)), which influence both the spatial and temporal haematocrit distribution in microvascular networks (Pop, 2007). In particular, the phase-separation (plasmaskimming) effect acting at divergent bifurcations (Pries et al., 1996), in which plasma is drawn down low-flux branches in preference to suspended red blood cells, is likely to influence strongly the flow environment in BEVs.

Features of individual BEVs have been elegantly described both ultrastructurally and also in an in situ model of juvenile rat mesentery (Rhodin and Fujita, 1989), where rouleaux (clusters of erythrocytes), platelet aggregation and circulatory stasis were recorded. As BEVs represent the leading edge of the primary vascular plexus and therefore regulate its initial patterning, knowledge of their structure, temporal development and physiological properties is integral to the understanding of how a primary plexus develops. Angiogenic sprouts, a proportion of which are BEVs, represent the first evidence of functional circulation at the leading edge of vascular growth into ischaemic tissue. New vessels, including BEVs, which have yet to form functional basement membranes or attract peri-vascular support cells, are characteristically leaky, allowing large molecular-weight proteins access to the interstitium. These proteins include platelet degranulation products (Italiano, 2008), matrix-degrading enzymes (Roy et al., 2006) and components of the haemostatic cascade, which serve as a scaffold for blood vessel ingress and wound healing (Laurens et al., 2006).

In this study, the temporal development of BEVs in relation to vascular closure in a small healing wound is presented and from this data a mathematical model relating geometry to function of these critical intermediary structures is proposed. Relatively few previous mathematical models of the microcirculation have considered flow in permeable vessels (Baish et al., 1997; Pozrikidis and Farrow, 2003); unlike these studies, the proposed model specifically considers nonuniform vessels that are closed at one end. The model enables quantification of the transport of plasma labelled with the passive tracer $150 \mathrm{kDa}$ FITC-dextran along the BEV and out into the interstitium, which in turn allows the permeability of the BEV walls to be determined. The model gives insight into the details of the transport properties that are inaccessible experimentally in situ. Combining theoretical and experimental techniques, the hypothesis that plasma skimming and vessel geometry determine the degree to which erythrocytes fill the BEV is investigated.

\section{Materials and methods}

\section{Animals and surgery}

Window-chamber surgery was performed as previously described (Lehr, 1993). Briefly, adult (10-12 week old) male CD1 mice (Harlan, UK) were anaesthetised with Hypnorm and Hypnovel and the hair on the dorsum was shaved with clippers. The skin was prepared for surgery by application of depilatory cream followed by 3 successive applications of Hibiscrub and Isopropanol. Titanium window chambers were implanted on the mid-dorsum and a small circular wound was created on the panniculus carnosus with a hot probe $(1 \mathrm{~mm}$ diameter, $70{ }^{\circ} \mathrm{C}$ for $3 \mathrm{~s}$ ). The preparation was imaged after injury with a Nikon Eclipse E600FN epi-fluorescent microscope using a variety of objective magnifications. Imaging of the vasculature was facilitated by the intra-venous (i.v.) injection of $50 \mu \mathrm{l}$ of $5 \% 150 \mathrm{kDa}$ FITC-dextran (Sigma-Aldrich, UK). A total of 3 regions of interest (ROIs) around the wound area were imaged, beginning at the surviving rim of the lesion. Successive images were taken at the leading edge of the vasculature (at higher magnifications) between days 5-8 when the greatest numbers of BEVs were observed.

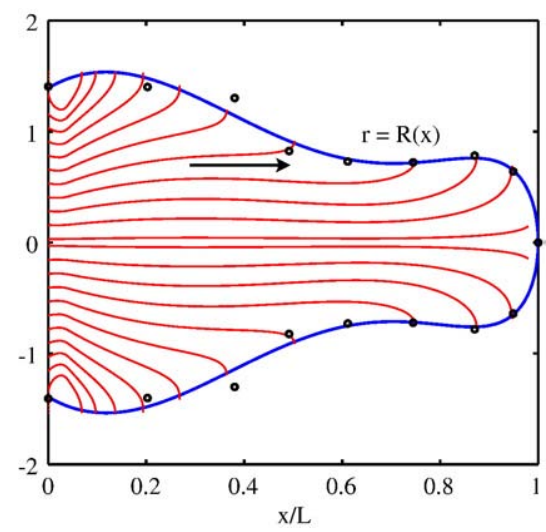

Fig. 2. Measurements of BEV radius $R(x)$ (scaled on the characteristic radius $R_{0}$ ) versus axial distance $x / L$ (points) fitted with a polynomial (solid, blue). Internal lines show streamlines, computed for $\alpha=1$. The flow as indicated by the arrow is from left to right 

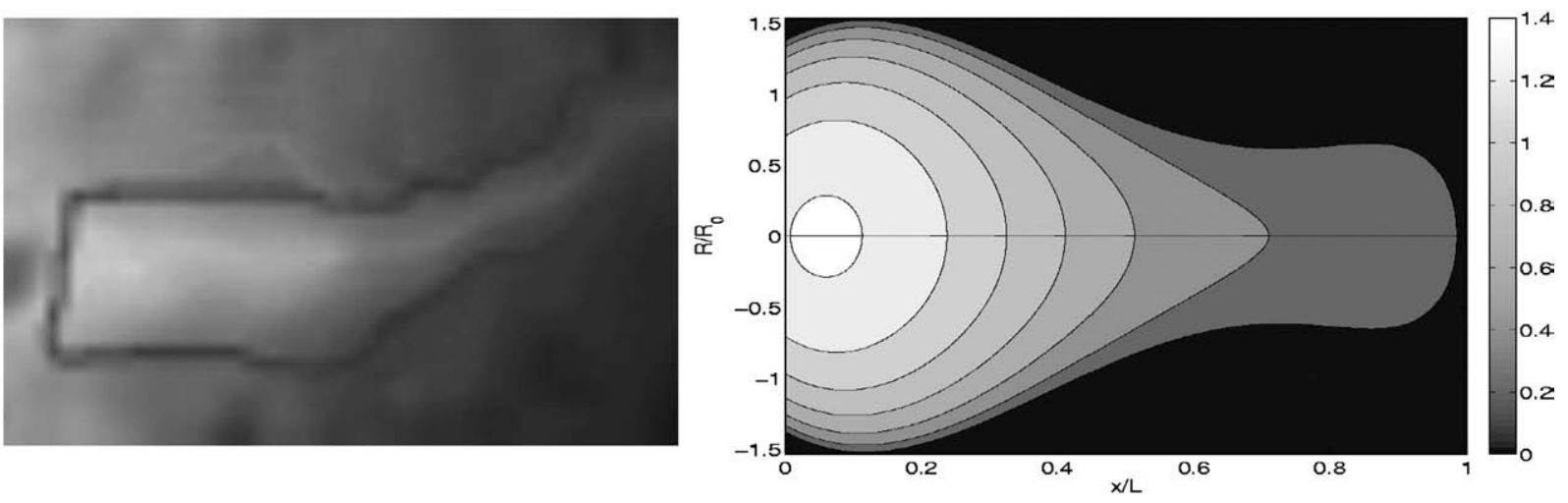

Fig. 3. Measured tracer intensity in a BEV outlined with a black line (left) and normalised computed tracer intensity (right, assuming $\alpha=0.01, \lambda=1.25$, Pe $=2$ ).

\section{Characterisation of blind-ended vessels}

After choosing the particular ROI, a 3-D image stack was prepared using IPLab (BD Biosciences: UK). BEVs were identified according to the following criteria: (i) there was no obvious circulation of erythrocytes through the vessel; (ii) there was a single parent vessel proximally, with definite features of flow; (iii) tracing of the candidate BEV through the 3-D series revealed no connection with any other vessel. BEVs were characterised either as plasma-filled, mixed (loosely stacked with erythrocytes) or plugged with erythrocyte rouleaux. Each image set was analysed by 2 independent observers and the results were tabulated and graphically represented. The lengths and diameters (minimum of 5 per BEV) were measured using IPlab and centreline intensity determined with an in-house programme (Fig. 1). The results of the analysis for each ROI were pooled for each animal/day and an average of the frequency of each type of BEV calculated \pm SEM.

\section{Segmentation of BEVs and parameter extraction}

Plasma-filled BEVs were traced and parameters used for the mathematical model were semi-automatically extracted using the Windows software VesselPro developed at the Systems Biology Research Group at the University of Ulster. This involved three manual processing steps. In a first step, the BEV is roughly outlined using a discrete B-spline curve, which can efficiently be evaluated by recursive filtering (Unser et al., 1993). The outline of the B-spline starts and ends at the attachment points of the BEV to its mother vessel (see Fig. 1A). To optimise the positions of each node of the B-spline curve around the BEV, a simple energy minimisation strategy (Kass et al., 1988) is employed to minimise the internal and external energies at each point (see Fig. 1B). After the B-spline is optimally fitted to the vessel, the underlying image is binarised and the inner line (the skeleton) is extracted using Rosenfeld's parallel thinning algorithm as described in (Cychosz, 1994). To ensure that the inner line starts in the middle of the attachment points of the mother vessel, an half circle is virtually added to the inlet diameter. On the opposite side, the inner line is extended along its final direction vector until it touches the boundary. This point is defined as the tip of the BEV. Final grey level values are then extracted by sampling at regular intervals along the extracted inner line and the values are written to an ASCII table that can be read by the simulation program. To reduce noise, grey levels were averaged over a $3 \times 3$ neighbourhood.

\section{Mathematical modelling}

A theoretical model for pressure-driven flow of plasma into a slender BEV was developed; mathematical details are given in the Appendix. Using segmented images of individual BEVs, the measured radius as a function of axial distance for each BEV was fitted with a polynomial function; an example is shown in Fig. 2. The length $L$ and characteristic radius $R_{0}$ (of the uniform cylinder of equivalent length and surface area) of each BEV was measured. This geometric information was inserted into the flow model, which treats each $\mathrm{BEV}$ as an axi-symmetric closed tube with permeable walls of nonuniform diameter, surrounded by interstitium. Exploiting the fact that $R_{0} \ll L$, the flow in the BEV is described using lubrication theory, a standard approximation for slow flow in slender tubes and channels (Acheson, 1990). Only plasma-filled BEVs (arising typically when
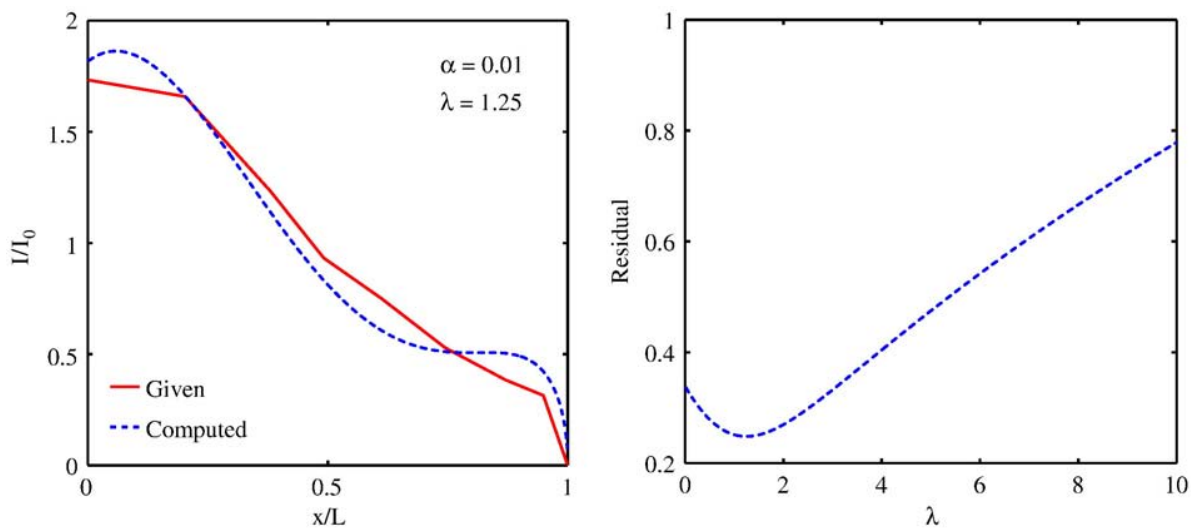

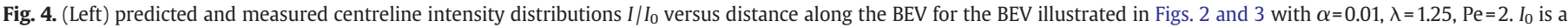

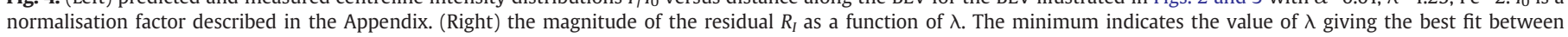
predicated and measured intensity distributions. 


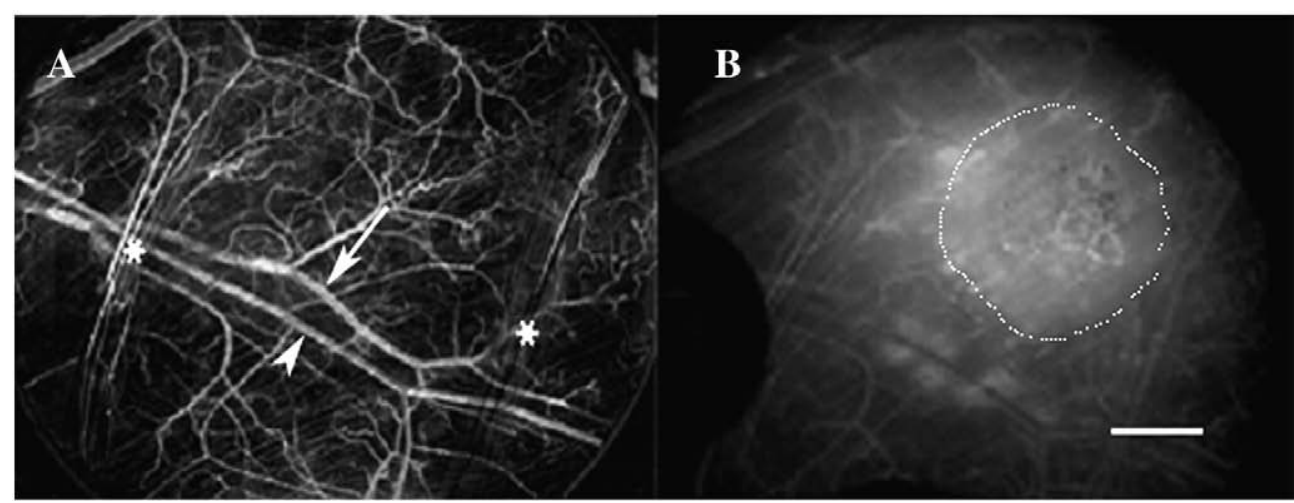

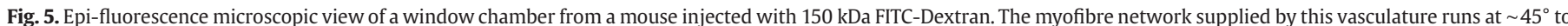

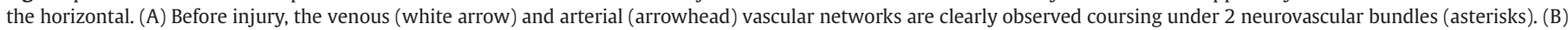

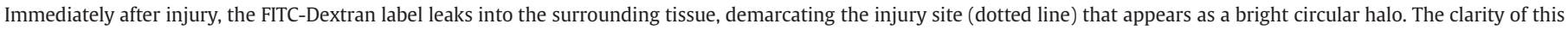
image is reduced due to the post-traumatic oedema. Scale bar=1 mm for both panels A and B.

$L \approx 10-100 \mu \mathrm{m}$ and $R_{0} \approx 2-5 \mu \mathrm{m}$ ) are modelled, treating plasma as an incompressible Newtonian fluid of viscosity $\mu \approx 0.016 \mathrm{~g} /(\mathrm{cm} \mathrm{s})$. Plasma can escape across the walls of the BEV at a rate determined by a filtration coefficient $\kappa$, assumed to be uniform along the BEV. It is also assumed that the flow of plasma is driven by $\Delta p$, the difference between the pressure at the BEV entrance and that in the interstitium (also assumed uniform). The model shows how the flow pattern in an individual BEV is characterised predominantly by the dimensionless filtration parameter $\alpha=16 \mu \kappa L^{2} / R_{0}^{3}$. This parameter, which measures the ratio of transmural to axial conductances, is very small compared to unity for normal vessels (when $R_{0} \approx 2 \mu \mathrm{m}, L \approx 10 \mu \mathrm{m}$ and $\kappa \approx 10^{-9} \mathrm{~cm}^{3}$ / (dyn s), $\alpha \approx 4 \times 10^{-5}$ ), indicating very little leakage. Streamlines illustrat- ing plasma flow inside a relatively leaky $\operatorname{BEV}(\alpha=1)$ are shown in Fig. 2.

To enable comparison with imaging, a model for transport of FITCdextran as a dilute passive solute was also created, using a simplified advection-diffusion equation (Friedman, 1986) for the cross-sectionally-averaged tracer concentration; this was possible due to the slender geometry of the BEV. Advective transport by plasma motion takes place along the BEV (at typical speeds we observed to be $U_{0}=5 \mu \mathrm{m} / \mathrm{s}$ ), across the BEV wall and into the interstitium. The tracer also diffuses (i) in the plasma (the Stokes-Einstein relation gives a diffusion coefficient $D \approx 3 \times 10^{-7} \mathrm{~cm}^{2} / \mathrm{s}$ for molecular radius $500 \mathrm{~nm}$ ), (ii) across the BEV wall (characterised by permeability parameter $\lambda_{C}$ )
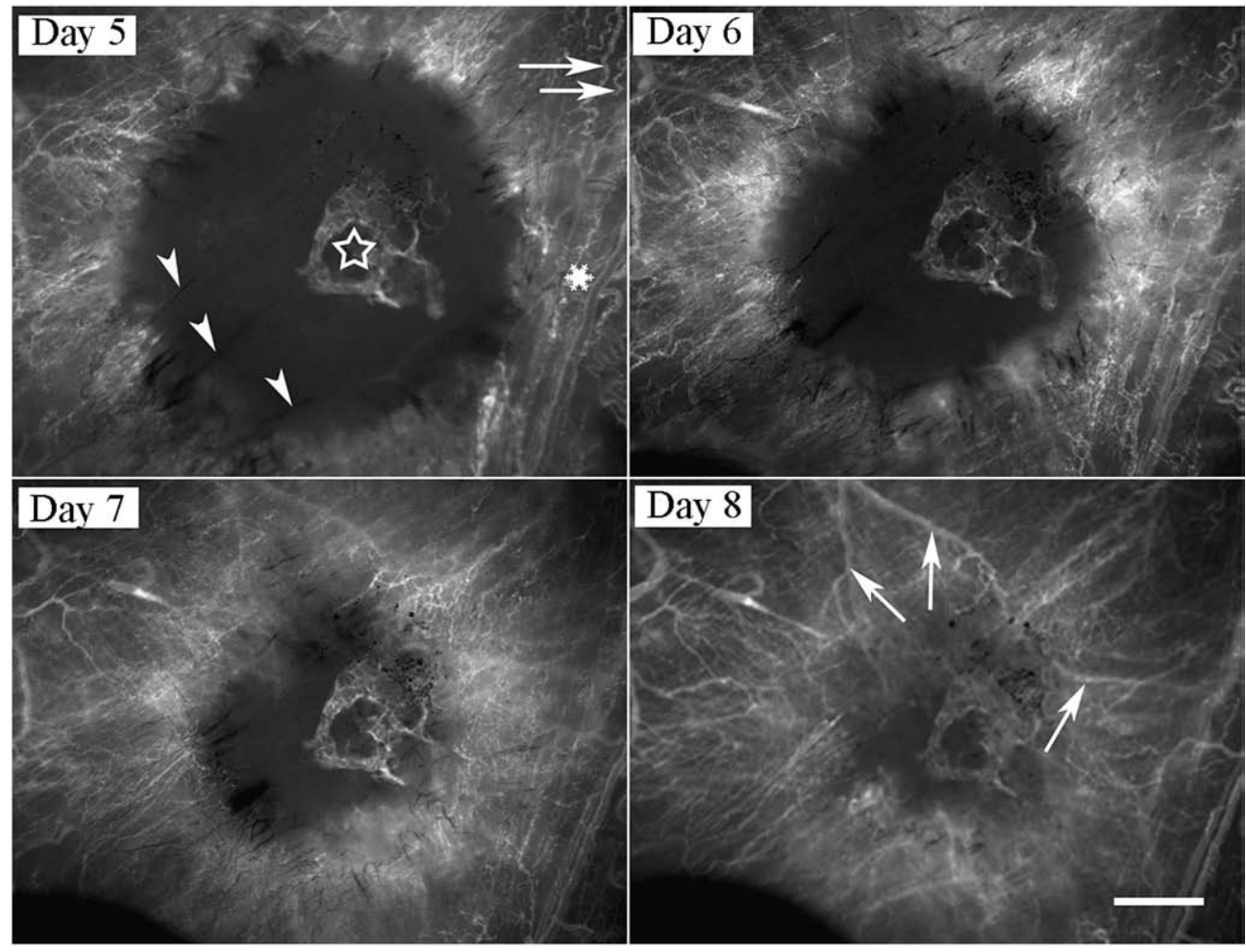

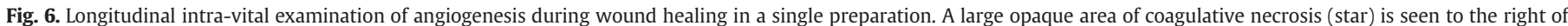

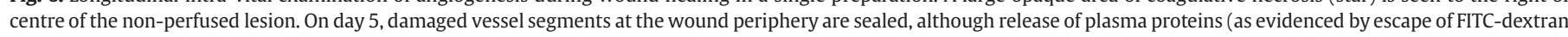

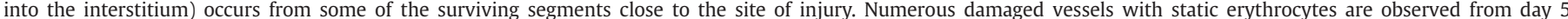

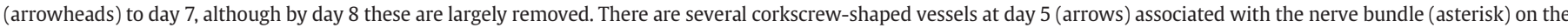

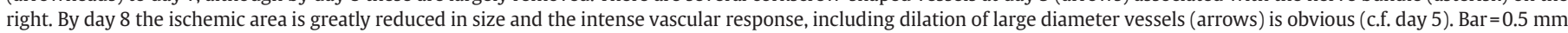
in all panels. 


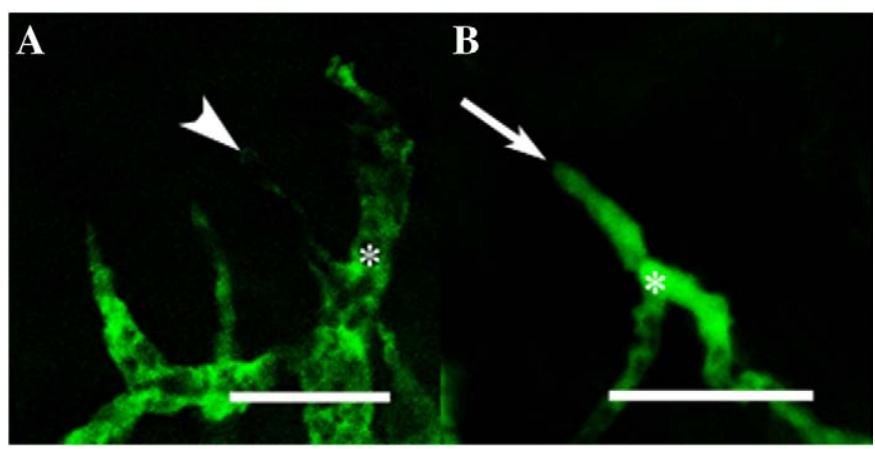

Fig. 7. Fluorescence microscopic images of $150 \mathrm{kD}$ FITC-dextran filled BEVs in a healing murine muscle wound at day 7. (A) BEVs at the leading edge of the angiogenic plexus have irregular morphologies, with inlet diameters exceeding $10 \mu \mathrm{m}$ (asterisk) and are occasionally filled with erythrocytes (arrowhead). (B) Plasma-filled BEVs (arrow) characteristically have a bright point of fluorescence at, or immediately adjacent to, the inlet (asterisk: cf. Fig. 3). Bars $=50 \mu \mathrm{m}$.

and (iii) in the interstitium. The relative importance of advective to diffusive transport along the vessel is measured by the dimensionless parameter $\mathrm{Pe}=U_{0} L / D$ (a Péclet number); using $R_{0} \approx 2 \mu \mathrm{m}$ and $L=10 \mu \mathrm{m}$, Pe is estimated at $\mathrm{Pe} \approx 2$, indicating that these effects are comparable. In contrast, diffusion in the interstitium (with coefficient $D_{\mathrm{i}} \approx 10^{-7} \mathrm{~cm}^{2} / \mathrm{s}$ (Fu et al., 2005) over length scales of order $L$ ) dominates advection, because the flow weakens by attenuation. The bulk permeability $D_{\mathrm{i}} /$ $L \approx 10^{-4} \mathrm{~cm} / \mathrm{s}$ is in series with a (typically) much smaller BEV wall permeability $\left(\lambda_{C}\right)$, so we hypothesise that the wall normally provides the dominant diffusive resistance. Thus, in addition to BEV shape and $\alpha$, which determine the plasma flow, tracer transport is characterised by the Péclet number Pe and the dimensionless permeability parameter $\lambda=L^{2} \lambda_{C} l\left(R_{0} D\right) .(\lambda \approx 2$ for parameters estimated as above, if we assume $\left.\lambda_{C}=1 \mu \mathrm{m} / \mathrm{s}\right)$.

Given a BEV shape (obtained experimentally), pressure and tracer distributions were computed for assumed values of $\alpha$, Pe and $\lambda$. An example of the solute distribution is shown in Fig. 3, alongside the corresponding image of a real BEV. Because the BEV is long and thin, the curvature of its centreline is assumed not to have a significant impact on the internal flow properties.

Integrating solute distributions across the plane containing the BEV axis, fluorescence centreline intensity distributions were predicted for a range of parameter values. Normalised predicted and measured centreline distributions were compared and best fits were used to infer levels of plasma leakage, by minimising a residual $R_{I}$ (defined in the Appendix). The fitting process for an individual BEV is illustrated in Fig. 4.

\section{Results}

Injury model

The viewing area in a window chamber ( $7.5 \mathrm{~mm}$ in diameter) is characterised by at least one arterio-venous bundle supplying the underlying striated muscle of the panniculus carnosus muscle and from 1-3 neurovascular bundles that course almost vertically through the chamber (Fig. 5A). The heat-induced injury was placed between these respective structures, taking particular care to minimise damage to neurovascular bundles, resulting in a circular lesion of approximately $2-2.5 \mathrm{~mm}$ in diameter (Fig. 5B). Although haemorrhage from the site was minimal at 15 min following injury, $150 \mathrm{kDa}$ FITC-dextran leakage into the interstitium was evident immediately following injury ( $\sim 5$ min: Fig. $5 B$ ). The edges of the lesion show the greatest fluorescence intensity, with a gradual diminution on either side of this rim (Fig. 5B), consistent with post-injury hyperemia (Langer, 2005).

\section{Plexus growth is led by BEVs}

The most intense period of angiogenesis occurred during days 5-8 (Fig. 6), although the first evidence of BEV formation was observed at day 3 (not shown). The lesion (as measured by the non-dextran perfused region) decreased in size daily and was accompanied by the inward radial growth of BEVs (Fig. 6), so that the entire lesion area was filled with vessels by day 15 (not shown). Corkscrew vessels, characteristic of alterations in regional perfusion following injury, were frequently observed close to the lesion area (Fig. 6). BEVs filled with plasma, partially filled with erythrocytes or rouleaux (erythrocyte columns; Fig. 7) were readily distinguished. The total numbers of BEVs (plasma-filled or containing erythrocytes) remained constant at around 70 per wound, from days 5-8 (Fig. 8A). BEV lengths were around $90 \mu \mathrm{m}$ on day 5 but were significantly shorter by days $6-8$ (Fig. $8 \mathrm{~B} ; p<0.001$ : repeated measures ANOVA). Furthermore, there was a clear correlation between length and luminal content of the BEVs (Table 1). Briefly, plasma-filled BEVs had significantly shorter mean lengths than those containing a few erythrocytes (mixed) or BEVs containing erythrocyte rouleaux, consistent with a plasma-skimming model (discussed below). Although single erythrocytes were occasionally observed entering BEVs (not shown), no estimates of the rate of entry were attempted experimentally, due to the low frequency of this occurrence. In addition, the lengths and diameters of individual
A

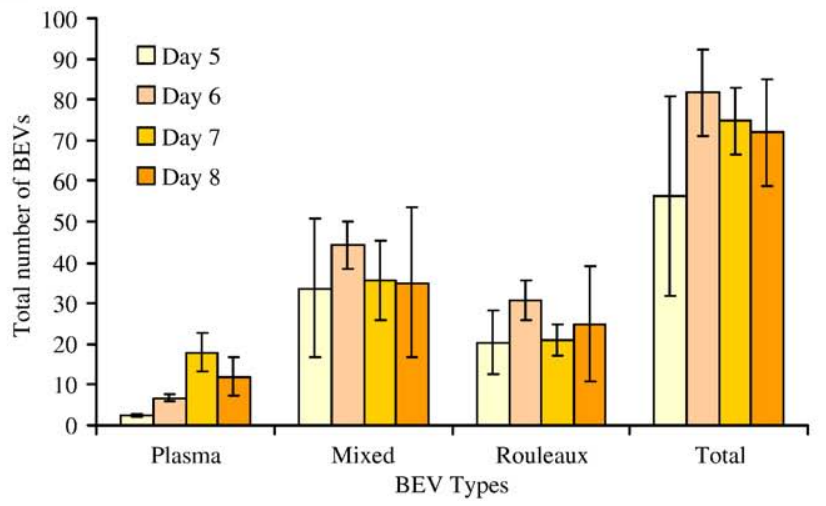

B

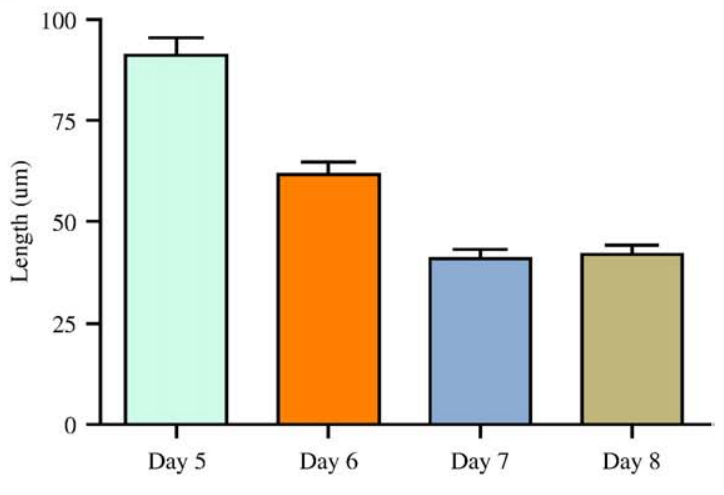

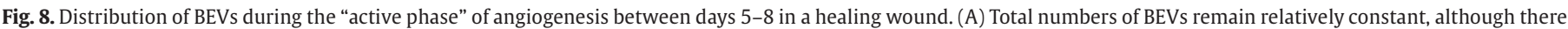

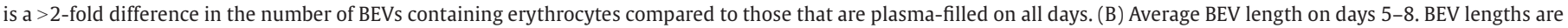

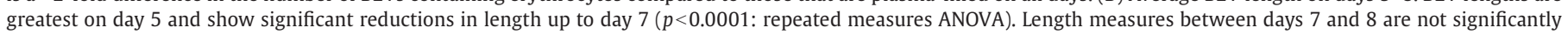
different $(p>0.05)$ 
Table 1

Longer BEVs tend to be filled with erythrocytes

\begin{tabular}{lllr}
\hline Day & Plasma & Mixed & \multicolumn{1}{l}{ Rouleaux } \\
\hline 5 & $41 \pm 13$ & $86 \pm 5(p=0.0039)$ & $105 \pm 7(p=0.0004)$ \\
6 & $37 \pm 7$ & $58 \pm 4(p=0.0038)$ & $74 \pm 5(p<0.0001)$ \\
7 & $21 \pm 2$ & $39 \pm 2(p<0.0001)$ & $63 \pm 5(p<0.0001)$ \\
8 & $19 \pm 2$ & $34 \pm 2(p<0.0001)$ & $65 \pm 5(p<0.0001)$ \\
\hline
\end{tabular}

Values in the table represent mean \pm SEM of BEV length $(\mu \mathrm{m})$. Values in brackets indicate significant differences (unpaired $T$-tests compared to the plasma-filled group on each day with a $95 \%$ confidence interval). Plasma-filled BEVs were significantly longer on day 5 compared with day 7 ( $p<0.05$; Bonferroni multiple comparison test).

BEVs on days 5-7 were measured in order to estimate the internal surface area of each BEV (Fig. 9), showing that BEVs with a larger surface area are more likely to contain erythrocytes, particularly on days 6 and 7 ( $p<0.05$, Mann-Whitney test with a 95\% confidence interval). Modelling estimates below suggest that plasma flux through BEVs is proportional to surface area, so that BEVs with a larger surface area will draw a greater flux of plasma from their parent vessel, increasing the likelihood of entraining erythrocytes and thereby becoming of the mixed type. From this we propose a plasmaskimming model for erythrocyte entry into vessels based on the BEV surface area, which is outlined below.

\section{Estimates of permeability}

The mathematical model was used to predict the flux of plasma into BEVs and tracer fluorescence intensity along them as functions of wall permeability and shape. The influence of varying the wall filtration parameter $\alpha$ is illustrated in the Appendix (Fig. 11). When filtration is weak $(\alpha \ll 1)$ a consistent pattern of behaviour is seen in which the plasma pressure is approximately uniform along the BEV, and the flux is given approximately by $\kappa \Delta p A$, where $A$ is the BEV

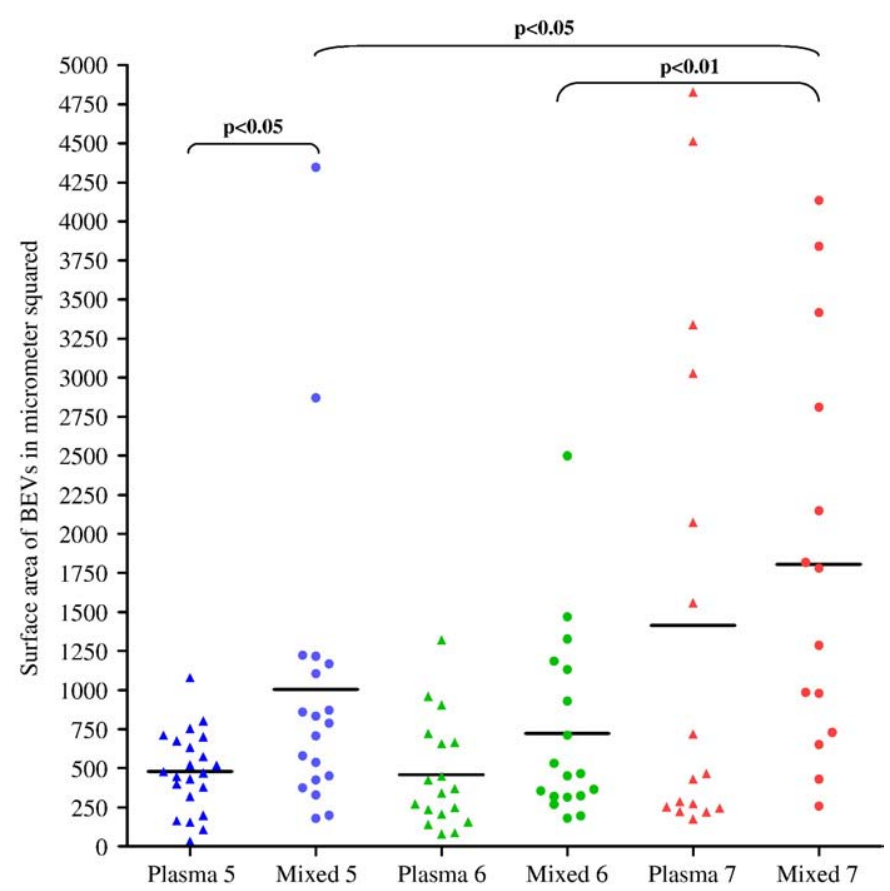

Fig. 9. Surface area of BEVs. BEVs were examined on individual days following injury and classified as filled with plasma alone or mixed (containing erythrocytes in their lumen). Length and diameters were measured and used to yield an estimate of internal surface area. BEVs containing erythrocytes had significantly larger surface area than plasma-filled vessels on day $5(p<0.05)$, while larger vessel surface areas were associated with erythrocyte ingress particularly between days 6-7 $(p<0.01)$ following injury; Mann-Whitney U-test.

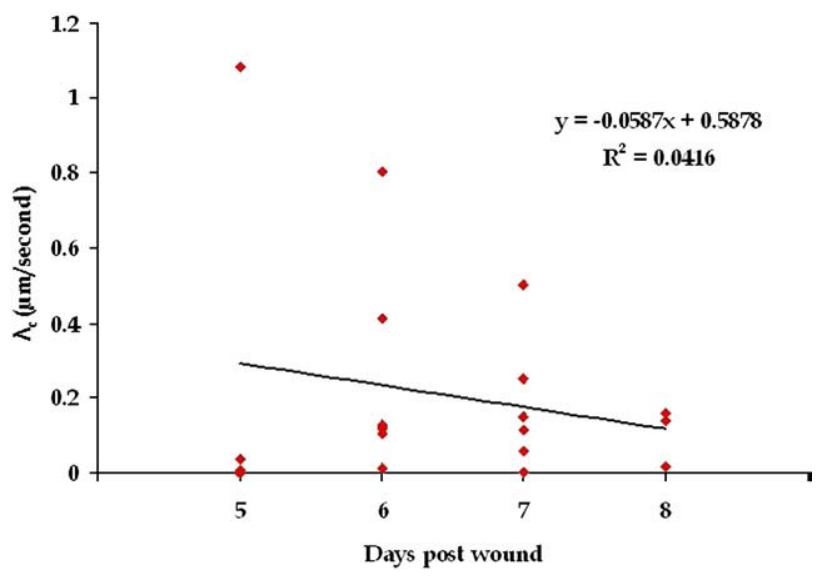

Fig. 10. Predicted BEV wall permeability $\left(\lambda_{c}\right)$ to $150 \mathrm{kDa}$ FITC-dextran versus the experimental day following wound induction. Using the mathematical model, the wall permeability was assessed as outlined in the appendix. Of the 36 data points presented in Fig. 9, 16 were discarded because the model did not predict an identifiable or sufficiently small residual (see Fig. 4 (right)). The remaining 20 data points are plotted here. Diamonds represent a calculation of $\lambda_{\mathrm{c}}$ for an individual BEV on the day indicated. The regression line shows a trend towards reduction in permeability of vessels over days $5-8$, although this is not statistically significant $(p>0.05)$.

surface area. We used $\alpha=0.01$ to describe plasma flow in this regime (this is representative of flow patterns for $\alpha<0.01$ ). Predicted tracer intensity distributions along the BEV centreline were then compared with measured intensities. Fig. 3 shows that there is good qualitative agreement between experimental data and theoretical predictions. Fig. 4 shows that good quantitative agreement between the predicted and measured intensity distributions can be achieved by choosing the best fit for the BEV wall permeability parameter $\lambda$. This best-fit parameter was evaluated for multiple BEVs in mice at days 5, 6, 7 and 8 . The best-fit (dimensional) permeability parameter $\lambda_{c}$ is presented in Fig. 10 and shows that there is a general reduction in the variation of vessel permeability from days $5-8$, although no statistically significant trend over days 5-8 can be established from this data set $(p>0.05)$. This reduction in vessel permeability variation has also been observed in our laboratory using Fluorescence Recovery After Photobleaching (FRAP) to analyse relative rates of flux across BEV walls (Machado, 2007).

\section{Discussion}

Vital imaging of vascular plexi is a long-established method for observation of angiogenesis both in healing wounds and during tumour development (Roberts and Lin, 2004). Many characteristic features of wound healing are observed in window chambers, such as hyperemia, angiogenesis and vascular remodelling, vascular stasis, vessel dilation or constriction and leukocytic cell infiltration. The major advantage of this method over histological, ultrastructural or biochemical analyses is that a range of data modalities (e.g., morphological, physiological and kinetic data) can be obtained in a sequential manner from individual wounds, allowing structurefunction hypotheses to be tested. In this study we follow the leading edge of angiogenic plexi in individual small $(\sim 2.0-2.5 \mathrm{~mm}$ in diameter) wounds contained within window chambers on the dorsum of mice. In the first hour following wounding of the panniculus carnosus muscle in the window chamber, vessel dilation and leakage of FITC-dextran near the tips of sealed vascular segments is observed, which is a characteristic early response to muscle injury (Scholz, 2003). Early induction of vasoactive agents adjacent to the injury serves to increase leakage of a variety of large molecular-weight proteins, which in this study was evident as intense FITC-dextran fluorescence at the rim of the injured area. The area immediately 
adjacent to the injury contains exudated fibrin (Laurens et al., 2006) and other filamentous proteins which form an early scaffold for angiogenic sprouts. In addition, matrix metalloproteases are released which loosen extracellular matrix interconnections (Roy et al., 2006) permitting the invasion of new blood vessels and other cell types involved in the wound healing response.

The predominant process of creating new vascular networks is via sprouting angiogenesis (Carmeliet and Jain, 2000), an invasive process involving the breakdown of the extracellular matrix by invading sprouts (Yana, 2007). Sprouts can be either solid cords composed of endothelial cells or blind-ended vessels (BEVs) which are intermediate structures in angiogenic loop formation (Rhodin and Fujita, 1989; Hashizume and Ushiki, 2002). Angiogenic sprouts contain specialised tip cells, which secrete matrix-degrading enzymes (Yana, 2007) allowing their invasion into the wound. We first observed capillary sprout formation on day 3 , in agreement with observations of the initiation of angiogenesis in several other experimental models of wound healing (Scholz, 2003; Phillips et al., 1991; Sephel et al., 1996; Wagatsuma, 2007). The initiation of the angiogenic response (on day 3 post-wounding, when edema and lesion size is maximal) is associated with an influx of macrophages and other inflammatory cells, which are derived principally from the vasculature (Langer, 2005). Further studies investigating rolling and adhesion phenomena should determine if BEVs are critical mediators of the influx of phagocytic cell types at the leading edge of the vascular plexus. The BEVs described herein represent only the proximally perfused segment of an advancing angiogenic sprout. Their cellular extensions, such as those described in the vasculature of the developing retina, may exceed $250 \mu \mathrm{m}$ in length (Gerhardt, 2003), providing the first templates and later circulatory conduits for spanning the edges of large lesions or avascular zones. Data from the present study support this hypothesis; the longest BEVs $(>150 \mu \mathrm{m})$ are observed at day 5 , when wound size is near maximal, with BEV numbers peaking between days 6-7 (Fig. 8A), when the most rapid decrease in wound area occur. By day 8 , when the wound size is markedly reduced, longer BEVs are rare and vessels $<40 \mu \mathrm{m}$ in length predominate.

In this study plasma-filled segments were found to be short structures $(<40 \mu \mathrm{m})$; however as surface areas increase, BEVs are more likely to contain erythrocytes than to be filled with plasma alone (see Fig. 9). One possible explanation for this observation is plasma skimming, embodied in the empirical phase-separation bifurcation law for flow in capillary networks (Pries et al., 1996); if the flux of plasma entering the BEV is sufficiently low, plasma adjacent to the wall of the parent vessel is drawn into the BEV without entraining any erythrocytes (Pries, 1989). In the limit of weak filtration $(\alpha \ll 1)$, which is most likely to be relevant to BEVs, our model shows that the plasma flux is given approximately by $\kappa \Delta p A$, where $A$ is the BEV surface area. Thus BEV surface area alone can be used to estimate plasma flux (assuming $\kappa$ and $\Delta p$ are fixed). When we relate the type of BEV (plasma only or mixed) to the surface area (Fig. 9), we observe a clear increase in number of mixed BEVs as this parameter increases. Provided a vessel is wide enough to admit a single erythrocyte, the threshold for plasma skimming is given in terms of the fractional plasma flux entering the daughter vessel exceeding a threshold dependent only on parent radius and haematocrit (Pries et al., 1996). Thus an increase in BEV length without an increase in radius is sufficient to increase surface area (and therefore flux) beyond the plasma-skimming threshold; this may explain why longer vessels are significantly more likely to contain erythrocytes (Table 1 ). Furthermore, plasma skimming may explain the clear longevity of the plasma-filled BEV population (Fig. 8A), in that BEVs with low permeability to plasma can remain plasma-filled even if they are wide enough to admit erythrocytes (Fig. 9). Likewise, plugging of BEVs with red cells may signal excessive leakiness. Since ingress of erythrocytes leads to vascular stasis, hypoxia, initiation of the haemostatic cascade and segment regression (Rhodin and Fujita,
1989), determining the rate at which BEVs become filled with erythrocytes could be a key indicator of a particular segments' survival. Testing of this hypothesis awaits further experimentation based on acute assessment of BEV development (from 0-24h after first observing an individual BEV).

Flow within the microvasculature has been extensively investigated in terms of the mechanical and rheological properties of the blood and its interaction with the vessel wall (reviewed in Popel and Johnson, (2005). Within vessels containing both an inlet and outlet, shear forces on the endothelial cells are key regulators of vascular segment survival (Meeson, 1996; Azmi and O'Shea, 1984). Low flow rates in EC cultures have been shown to result in alterations in the distribution of cytoskeletal proteins (Dardik, 2005) and morphology (Malek, 1993) and induction of tissue factor expression, the cell surface initiator protein for the haemostatic cascade. In addition, cultures exposed to physiologically low rates of flow yield gene expression profiles similar to endothelium from atherosclerotic-prone arteries (e.g. carotid bifurcations) (Dardik, 2005; Brooks et al., 2004; Resnick, 1997; Wasserman and Topper, 2004) and also show higher levels of endothelial apoptosis (Tricot, 2000). In the present study a model is proposed for a unique vascular structure, in which there is a circulatory input (visualised by 150 kDa FITC-dextran) but very low flow and therefore the shear stress is also low. Significant shear stress will only be induced on ECs lining the walls of BEVs following arcade formation that occurs in this in vivo model in a period of less than $24 \mathrm{~h}$. Thus, we would hypothesise that, as time progresses, a larger proportion of EC within the walls of these BEVs become apoptotic due to the lack of physiological shear stress: testing this awaits the development of an appropriate in vivo fluorescent probe.

We have developed a mathematical model that is specific for BEVs, where a parent vessel provides the inflow (see Figs. 1 and 7) and the endothelial wall provides the barrier to flux. BEVs have been shown to be transient features, which are incorporated into the rapidly growing network, and that length distributions change during temporal progression in a healing wound. An understanding of the relationship between the morphological features of vessel segments having only an inlet and how the flux across the vessel wall regulates flow into them will provide important insights into the development of angiogenic plexi. We described the morphological dimensions of BEVs in a healing wound and measured the centreline intensity of FITC-dextran-labelled tracer, in addition to developing a mathematical modelling of plasma and tracer transport along the BEV and across the vessel wall (exploiting the geometrical data obtained experimentally). We characterised the leakiness of the BEV through a parameter $\alpha$, which is proportional to the mean wall shear stress in a closed-ended permeable vessel relative to that in an open-ended vessel of the same average radius and length (see the Appendix). Observations of flow speeds show that $\alpha \ll 1$ in BEVs, implying shear stresses in leaky BEVs are substantially smaller than those in patent capillaries of equivalent diameter and length.

The mathematical modelling of the biomechanical environment of BEVs has provided some insights beyond those available by imaging alone. During the period of most intense angiogenesis (days 5-8), flux of $150 \mathrm{kDa}$ FITC-dextran across the vessel wall is dramatically reduced as measured by in situ Fluorescence Recovery After Photobleaching (FRAP: data not shown). Recovery of fluorescence intensity inside BEVs on day 5 was highly variable, whereas by day 7, the recovery of post-bleach levels was essentially instantaneous. These findings indicate that BEVs on day 7 are functionally more mature than those on day 5 (Machado and Mitchell: unpublished observations), similar to the predictions reported in Fig. 10. In addition to drawing the important connection between BEV surface area and flux as discussed above, and the possible association with plasma skimming, we were able to use the model to predict the centreline distribution of a fluorescent tracer and hence to show that the permeability of the BEV population to tracer showed a decrease in variation over time. 
Following wounding or burns, a matrix-rich network of fibres is laid down which promotes angiogenesis. During this process a rapid and intense neovascularisation, partially controlled by angiogenic growth factors such as FGF-2 and VEGF (Brown, 1992; Nissen, 1998), results in greatly increased vascular density within the healing wound (Swift et al., 1999). Subsequently, matrix and tissue reorganisation lead to the pruning of the vascular network to suit the metabolic demands of the healing wound. Precisely which vessel segments regress or survive in individual wounds is largely unexplored; however, in situ imaging, coupled with mathematical modelling of data from these longitudinal analyses, has the potential to reveal the functional (flow and shear stress) characteristics and the vascular morphological and molecular (e.g. apoptotic) parameters that regulate this phenomenon and regulate vascular pattern formation in vivo.

\section{Acknowledgment}

This work was supported by EPSRC grant number GR/T19599/01.

\section{Appendix A. Supplementary data}

Supplementary data associated with this article can be found, in the online version, at doi:10.1016/j.mvr.2008.06.005.

\section{References}

Acheson, D.J., 1990. Elementary Fluid Mechanics. Oxford Applied Mathematics and Computing Science Series. Oxford University Press.

Azmi, T.I., O'Shea, J.D., 1984. Mechanism of deletion of endothelial cells during regression of the corpus luteum. Lab. Invest. 51 (2), 206-217.

Baish, J.W., Netti, P.A., Jain, R.K., 1997. Transmural coupling of fluid flow in microcirculatory network and interstitium in tumors. Microvasc. Res. 53 (2), 128-141.

Brooks, A.R., Lelkes, P.I., Rubanyi, G.M., 2004. Gene expression profiling of vascular endothelial cells exposed to fluid mechanical forces: relevance for focal susceptibility to atherosclerosis. Endothelium 11 (1), 45-57.

Brown, L.F., et al., 1992. Expression of vascular permeability factor (vascular endothelial growth factor) by epidermal keratinocytes during wound healing. J. Exp. Med. 176 (5), 1375-1379.

Burri, P.H., 1992. Intussusceptive microvascular growth, a new mechanism of capillary network formation. Exs 61, 32-39.

Carmeliet, P., Jain, R.K., 2000. Angiogenesis in cancer and other diseases. Nature 407 (6801), 249-257.

Cychosz, J.M., 1994. Efficient binary image thinning using neighborhood maps. In: Heckbert, P.S. (Ed.), Academic Press Graphics Gems Series. Academic Press Professional, San Diego, CA, pp. 465-473.

Dardik, A., et al., 2005. Differential effects of orbital and laminar shear stress on endothelial cells. J. Vasc. Surg. 41 (5), 869-880.

Friedman, M., 1986. Principles and Models of Biological Transport. Springer-Verlag. Berlin.

Fu, B.M., Adamson, R.H., Curry, F.R., 2005. Determination of microvessel permeability and tissue diffusion coefficient of solutes by laser scanning confocal microscopy. J. Biomech. Eng. 127 (2), 270-278.

Gargett, C.E., Rogers, P.A., 2001. Human endometrial angiogenesis. Reproduction 121 (2), 181-186.

Gerhardt, H., et al., 2003. VEGF guides angiogenic sprouting utilizing endothelial tip cell filopodia. J. Cell. Biol. 161 (6), 1163-1177.

Hanahan, D., Folkman, J., 1996. Patterns and emerging mechanisms of the angiogenic switch during tumorigenesis. Cell 86 (3), 353-364.

Hashizume, H., Ushiki, T., 2002. Three-dimensional cytoarchitecture of angiogenic blood vessels in a gelatin sheet implanted in the rat skeletal muscular layers. Arch. Histol. Cytol. 65 (4), 347-357.
Italiano Jr., J.E., et al., 2008. Angiogenesis is regulated by a novel mechanism: pro- and anti-angiogenic proteins are organized into separate platelet \{alpha\}-granules and differentially released. Blood 111 (3), 1227-1233.

Kass, M., Witkin, A., Terzopoulos, D., 1988. Snakes: active contour models. Int. J. Comput. Vis. 1 (4), 321-331.

Kopp, H.G., Ramos, C.A., Rafii, S., 2006. Contribution of endothelial progenitors and proangiogenic hematopoietic cells to vascularization of tumor and ischemic tissue. Curr. Opin. Hematol. 13 (3), 175-181.

Langer, S., et al., 2005. New model for in vivo investigation after microvascular breakdown in burns: use of intravital fluorescent microscopy. Burns 31 (2), 168-174.

Laurens, N., Koolwijk, P., de Maat, M.P., 2006. Fibrin structure and wound healing. J. Thromb. Haemost. 4 (5), 932-939.

Lehr, H.A., et al., 1993. Dorsal skinfold chamber technique for intravital microscopy in nude mice. Am. J. Pathol. 143 (4), 1055-1062.

Machado, M., et al., 2007. Longitudinal analysis of plasma-filled, blind ended vesse sprouts at the leading edge of an angiogenic plexus. Microcirculation 14 (4 [IMG5]), 439-569.

Malek, A.M., et al., 1993. Fluid shear stress differentially modulates expression of genes encoding basic fibroblast growth factor and platelet-derived growth factor B chain in vascular endothelium. J. Clin. Invest. 92 (4), 2013-2021.

Meeson, A., et al., 1996. A relationship between apoptosis and flow during programmed capillary regression is revealed by vital analysis. Development 122 (12), 3929-3938.

Nissen, N.N., et al., 1998. Vascular endothelial growth factor mediates angiogenic activity during the proliferative phase of wound healing. Am. J. Pathol. 152 (6), 1445-1452.

Phillips, G.D., Whitehead, R.A., Knighton, D.R., 1991. Initiation and pattern of angiogenesis in wound healing in the rat. Am. J. Anat. 192 (3), 257-262.

Popel, A.S., Johnson, P.C., 2005. Microcirculation and hemorheology. Annu. Rev. Fluid Mech. 37, 43-69.

Pop, S.R., et al., 2007. Shock formation and non-linear dispersion in a microvascular capillary network. Math Med. Biol. 24 (4), 379-400.

Pozrikidis, C., Farrow, D.A., 2003. A model of fluid flow in solid tumors. Ann. Biomed. Eng. 31 (2), 181-194.

Pries, A.R., et al., 1989. Red cell distribution at microvascular bifurcations. Microvasc Res. 38 (1), 81-101.

Pries, A.R., Secomb, T.W., Gaehtgens, P., 1996. Relationship between structural and hemodynamic heterogeneity in microvascular networks. Am. J. Physiol. 270 (2 Pt. 2), H545-H553.

Resnick, N., et al., 1997. Endothelial gene regulation by laminar shear stress. Adv. Exp. Med. Biol. 430, 155-164.

Rhodin, J.A., Fujita, H., 1989. Capillary growth in the mesentery of normal young rats Intravital video and electron microscope analyses. J. Submicrosc. Cytol. Pathol. 21 (1), 1-34.

Risau, W., Flamme, I., 1995. Vasculogenesis. Annu. Rev. Cell Dev. Biol. 11, 73-91.

Roberts, R.L., Lin, P.C., 2004. Structural and functional optical imaging of angiogenesis in animal models. Methods Enzymol. 386, 105-122.

Roy, R., Zhang, B., Moses, M.A., 2006. Making the cut: protease-mediated regulation of angiogenesis. Exp. Cell. Res. 312 (5), 608-622.

Scholz, D., et al., 2003. Angiogenesis and myogenesis as two facets of inflammatory post-ischemic tissue regeneration. Mol. Cell. Biochem. 246 (1-2), 57-67.

Sephel, G.C., Kennedy, R., Kudravi, S., 1996. Expression of capillary basement membrane components during sequential phases of wound angiogenesis. Matrix Biol. 15 (4) 263-279.

Swift, M.E., Kleinman, H.K., DiPietro, L.A., 1999. Impaired wound repair and delayed angiogenesis in aged mice. Lab. Invest. 79 (12), 1479-1487.

Tricot, O., et al., 2000. Relation between endothelial cell apoptosis and blood flow direction in human atherosclerotic plaques. Circulation 101 (21), 2450-2453.

Unser, M., Aldroubi, A., Eden, M., 1993. B-spline signal processing: part II - efficient design and application. IEEE Trans. Signal Process. 41 (2), 834-848.

Vogeli, K.M., et al., 2006. A common progenitor for haematopoietic and endothelial lineages in the zebrafish gastrula. Nature 443 (7109), 337-339.

Wagatsuma, A., 2007. Endogenous expression of angiogenesis-related factors in response to muscle injury. Mol. Cell. Biochem. 298 (1-2), 151-159.

Wasserman, S.M., Topper, J.N., 2004. Adaptation of the endothelium to fluid flow: in vitro analyses of gene expression and in vivo implications. Vasc. Med. 9 (1), 35-45.

Yana, I., et al., 2007. Crosstalk between neovessels and mural cells directs the sitespecific expression of MT1-MMP to endothelial tip cells. J. Cell. Sci. 120 (Pt. 9), $1607-1614$ 